\title{
Fast switching pneumatic valves: experimental bench for flow and pulsed air jet characterizations
}

\author{
T. Ferreira, S. Sesmat, E. Bideaux, F. Sixdenier \\ Laboratoire Ampère, Université de Lyon, Lyon, France \\ E-mail: Thiago.caetano-ferreira@insa-lyon.fr, Sylvie.sesmat@insa-lyon.fr, Eric.bideaux@insa-lyon.fr, \\ Fabien.sixdenier@univ-lyon1.fr
}

\begin{abstract}
This paper presents a new experimental bench which allows the characterization of different fast switching valves in terms of flow rate and free air jet generation. By using this new bench, it is possible to study the different phenomena that may occur in the inner and outer circuits from the source up to the free jet. The different sensors positioned at key points along the air circuit allow the determination of the flow characteristics as well as the dynamic behavior of the different components during the generation of the pulsed air jets. By the tests performed with a fast spool-sleeve valve, it was possible to determine its flow characteristics (sonic conductance and critical pressure ratio). These values were used to build an average mass flow rate curve which is compared to the one measured by using a flowmeter during dynamic tests. This comparison enables to evaluate the limit frequency up to which the steady state flow characteristics can be used to estimate the average mass flow rate during dynamic operation. The next step consists in exploring the jet in a 3D space by using a 5-hole probe which provides the information on pressure, temperature and velocity necessary to determine the most suitable zone to perform object sorting with the jet. Also its unsteady behavior can be observed allowing the identification of the limit frequency at which the jet efficiency starts to decrease.
\end{abstract}

Keywords: Fast switching, pneumatic valve, pulsed air jet, mass flow rate characterization

\section{Introduction}

The development of new fast switching pneumatic devices is an important challenge for the fluid power industry [1][2][3]. The use of these elements on power transmission provides benefits in terms of power density, compactness, possibility to work in harsh environments, reliability and low cost.

The market for fast switching pneumatic valves with high flow capacity is in growth. Most manufacturers offer these components for pulsed air jets generation and the applications can be in the automotive industry, food processing, sorting elements and so forth. They also announce their products can achieve response time on the order of a few milliseconds, which leads to the generation of jets at frequencies up to $1 \mathrm{kHz}$, for flow rates about 150 $\mathrm{Nl} / \mathrm{min}$ (ANR).

First of all, for the food processing and sorting applications, the distance between the generated jets is highly constrained in a way the sorting operation can be precisely executed. Due to this fact, the miniaturization of the valve is also a major problem for these components, once the product must keep the small dispersion on response time and flow, good energy efficiency and long lifetime.

Also to perform the sorting operation it is necessary to know the jet coming from the valve and understand how is it affected by the dynamic behavior of the mechanical elements.

Some theoretical and simulation studies have been already carried out [4][5] and present theories and analytical formulation for air jets under certain conditions. However, steady state and dynamic behaviors of the whole circuit (valve, inner and outer circuit and free air jet) are being studied recently. [6][7][8][9].

Motivated by this problematic, an experimental bench was designed and built in order to evaluate the mechanical and pneumatic performances on air jet generation by fast switching valves. This bench enables the evaluation of the flow along the entire path from the source up to the free air jet and under different working conditions. 


\section{Objective}

This paper presents the experimental bench designed to study the mechanical and pneumatic behavior of the system used in sorting applications. The steady state flow characteristics of a system composed by an inner circuit, fast switching valve and nozzle can be determined with no need of disassembling the different elements. In a same way, the study of their performances in dynamic applications can also be performed.

From the steady state mass flow rate characteristics of the valve, and its dynamic response, it becomes possible to determine the average mass flow rate according to the working frequency of the system.

The analysis carried out enables the determination of the mechanical limitation of the system as well as the pneumatic behavior of the air flow in the inner circuit.

Also, a first study of a steady jet is presented. Its complete characterization using a multi-hole probe will be performed in the next steps and also the influence of the system's dynamics on pulsed air jet generation.

\section{Experimental procedure}

\subsection{Test bench}

The experimental bench was developed in a way to study and understand the different phenomena occurring along the flow path from the source up to the free jet. The bench is presented in Figure 1.

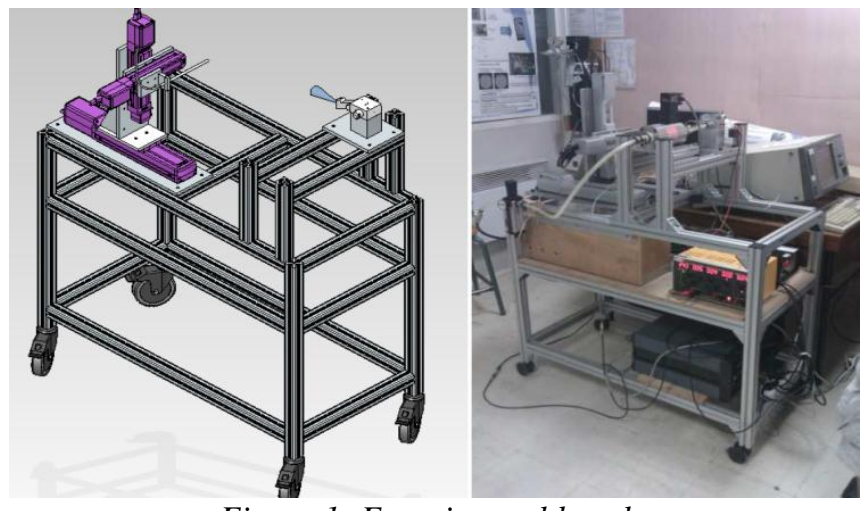

Figure 1: Experimental bench.

The pressures along the circuit upstream to the nozzle are measured using miniature pressure sensors to be able to measure dynamic pressure with a high bandwith. In order to obtain the position of the pneumatic shutter of the valve, a magnetic displacement sensor is used. The average air mass flow rate is measured by a mass flowmeter placed between the inlet and a tank used to filter the pressure oscillations induced by the frequency operation of the valve.

A 3-axis robot is used to precisely move a 5-hole probe (Figure 2), designed by Aeroprobe [10]. This enables the determination of the total and static pressures, total temperature and velocity (module and orientation) at different points in the free air jet. It is done by measuring the pressures and temperature at the tip of the probe. [11][12]

This device allows having a better understanding of the jet in order to optimize the energy required to eject different objects by using a pulsed air jet.
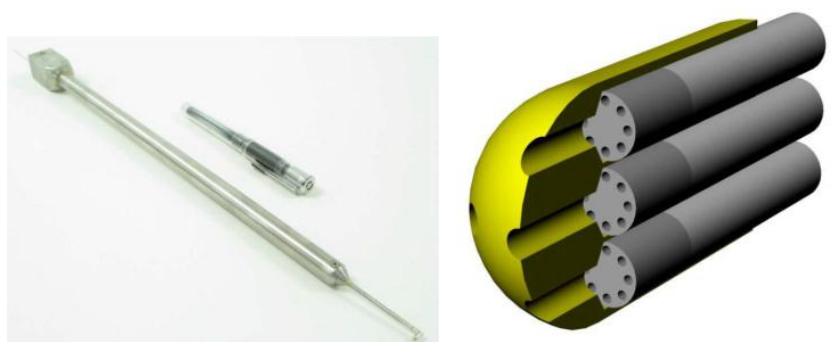

Figure 2: 5-Holes probe [10]

\subsection{Steady State characterization}

The scheme in Figure 3 shows the complete pneumatic circuit and the location of the sensors.

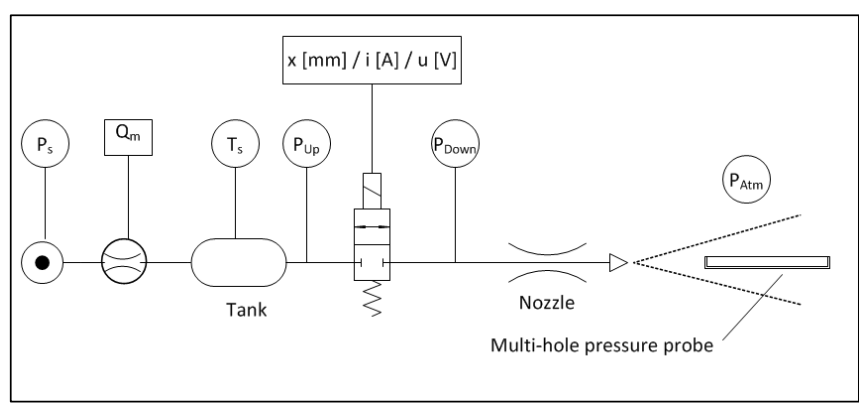

Figure 3: Pneumatic circuit scheme

In order to determine the flow rate characteristics of the different parts of the circuit upstream to the nozzle, it is necessary to build for each part the pneumatic conductance curve function of the downstream to upstream pressure ratio. Considering the flow as being adiabatic, the conductance can be calculated from the measurement of steady state mass flow rate, pressures upstream and downstream the element and temperature measured at the source $T_{s}$ (equation 1).

$$
C_{e}\left(\frac{P_{d}}{P_{u}}\right)=\frac{q_{m}\left(\frac{P_{d}}{P_{u}}\right)}{\rho_{0} P_{u}} \sqrt{\frac{T_{s}}{T_{0}}}
$$

Usually the sonic conductance and the critical pressure ratio of the valve are determined in steady state flow for the completely opened flow section according to ISO 6358 standard [13]. In this way, during simulations of pneumatic behavior of solenoid valves, it is of common sense to use, for intermediate positions, a conductance value proportional to the sonic conductance by a factor $\mathrm{x}(0<\mathrm{x}<=1))$ which takes into account the intermediate flow sections. The 
critical pressure ratio is supposed to be constant for all positions.

In this particular studied valve, due to its functioning way and the present sensors, it is possible to obtain one flow rate measurement for each intermediate position of the spool, which leads to intermediate points for the conductance curve.

In order to complete the steady state characterization, measurements with the 5-Holes probe were performed in a steady jet issued from the system.

\subsection{Dynamic characterization}

As the valve is used to generate pulsed jets, the understanding of its dynamic behavior is mandatory. In such mechanical systems different phenomena may occur (resonance, blocking, shocks) which may lead to a misuse and degradation of their performance. It is expected that these events have a direct influence on the pulsed jet generation. However, the real relation has yet to be determined.

Assuming that the flow rate is instantaneously established the dynamic mass flow rate $q_{m}(t)$ for a given time, can be calculated using the steady state flow characteristics depending on the mobile part position $x(t)$ with equation 2 [14].

$$
q_{m}(t)=C(x(t)) \cdot \rho_{0} \cdot P_{u}(t) \sqrt{\frac{T_{0}}{T_{s}}} \cdot\left(1-\left(\frac{\frac{P_{d}}{P_{u}}(t)-b(x(t))}{1-b(x(t))}\right)^{2}\right)^{m}
$$

By integrating $q_{m}(t)$ on one cycle, one can obtain the average mass flow rate $Q_{m}$ flowing through the valve and compare it with the measurement performed by the mass flowmeter after stabilization for a given frequency.

\section{Results}

\section{1 - Steady state characterization}

The tests were performed with a 2 stage spool and sleeve valve. The opening operation is performed by turning its electromagnetic actuator on, which allows the air to flow and push a spool inside a sleeve opening the flow section of the valve. To close, the electric circuit is turned off and the spool is returned to its initial position by means of a flat spring.

The displacement of the spool is measured as well as the voltage and current on the electrical circuit of the actuator. Also the mass flow rate is obtained by using the mass flowmeter as shown in Figure 3.

First of all, in order to identify the working pressure range of the valve first measurements were performed. In this test, the upstream pressure $P_{U p}$ was progressively increased whilst the spool displacement and the flow rate were measured. The resulting curve is presented in Figure 4.

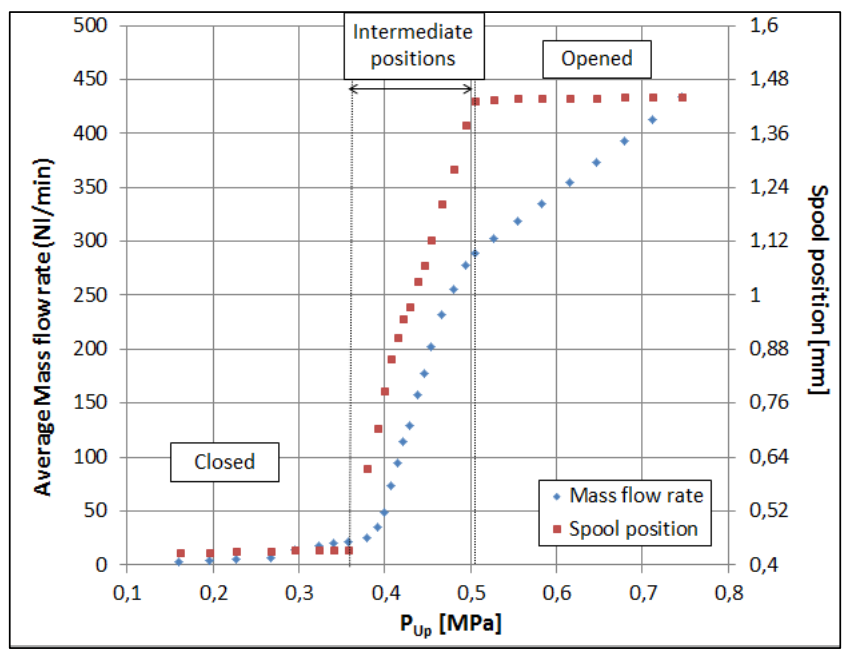

Figure 4: Flow rate and spool position function of the upstream pressure $P_{U p}$.

Three different zones can be distinguished in this figure. Up to an upstream absolute pressure of $0,35 \mathrm{MPa}$ the valve remains closed. The slight rising on the flow rate is due to the functional leakage of the system. From 0,35 to 0,5 MPa, the spool is in an intermediate position and the flow section is not completely opened. Above the pressure of $0,5 \mathrm{MPa}$ the spool reaches its maximum displacement and the flow increases proportionally to the upstream pressure.

For the steady state characterization of the components present in the circuit, the upstream pressure $P_{S}$ is set in intermediate values up to $0,7 \mathrm{MPa}$ to vary the pressure ratios. During the tests, different pressures along the circuit are measured (see Figure 3). From the values obtained the different conductance curves of the components can be drawn according to equation 1 .

In Figure 5 an unusual conductance curve of the valve is presented. In a similar way as in Figure 4, it is possible to identify 3 different zones. The first points (red diamond shaped) represent the rising upstream pressure with the valve still closed. As the pressure keeps rising whilst the spool still in its initial position, we observe a saturation behavior which could indicate a chocked leakage flow even before its opening. This hypothesis seems not to be equivocal once the minimal pressure to open the component is already at $0,35 \mathrm{MPa}$.

Another important zone is the points with maximum opening where the flow is certainly chocked (purple circles) once they all have the same conductance and pressure ratio values. The pressure ratio is blocked to its critical value whatever is the upstream pressure. 


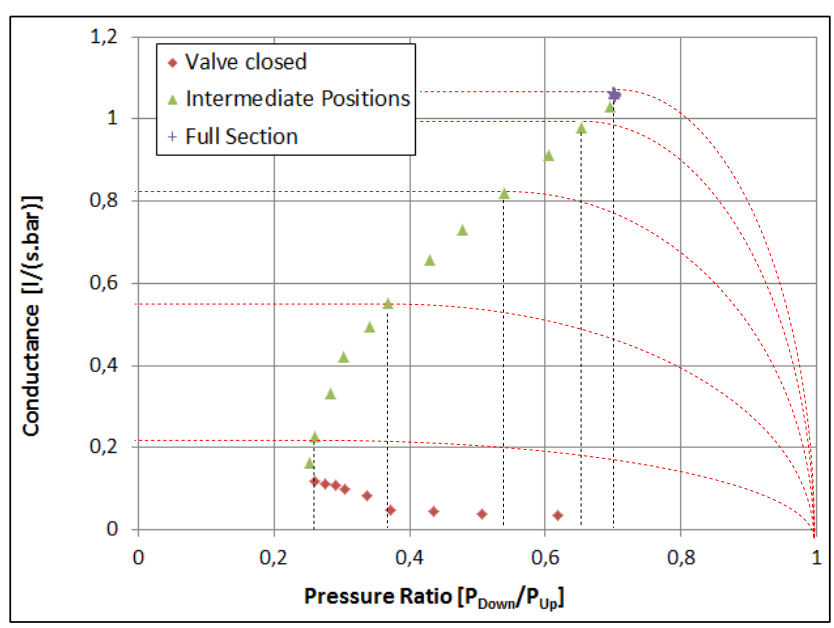

Figure 5: Measured Conductance curve of the Valve for different positions $\left(P_{U p}\right.$ to $\left.P_{\text {Down }}\right)$.

These facts lead to a strong hypothesis: considering all the intermediate points as being critical flows for intermediate flow sections (see dotted lines in Figure 5).

Due to the assumptions, it is then possible to determine a relation of the sonic conductance (Figure 6a) and critical pressure ratio of the valve (Figure $6 \mathrm{~b}$ ) for different spool positions. Using these curves and having the position and pressure values measured during the dynamic tests, it is possible to recover the information about the dynamic mass flow rate and its average value for one cycle during its operation.

The experimental conductance curve for the entire pneumatic circuit from the valve upstream pressure up to atmosphere is presented in the Figure 7. Its unusual shape comes logically from the flow section variation of the valve according to the circuit upstream pressure. Also the three zones already discussed for the valve (functional leakage progressive opening - full opening) appear clearly.

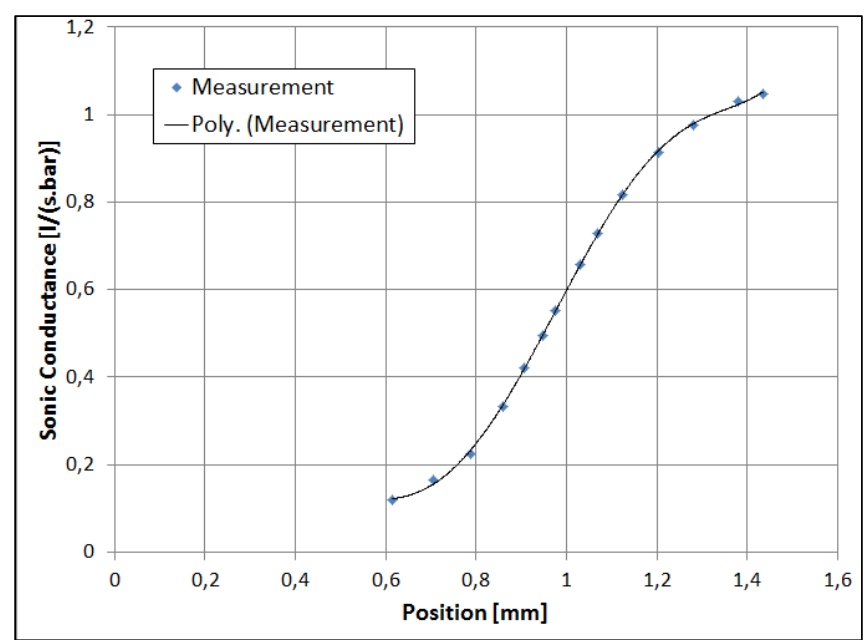

a)

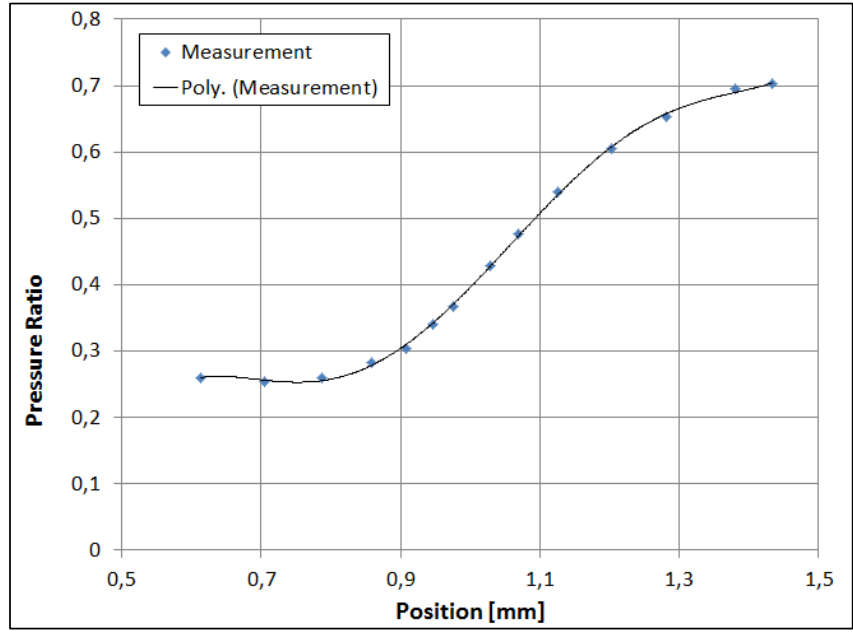

b)

Figure 6: Measured flow characteristics of the valve for intermediate positions and their polynomial approximation a) Sonic conductance; b) Critical pressure ratio.

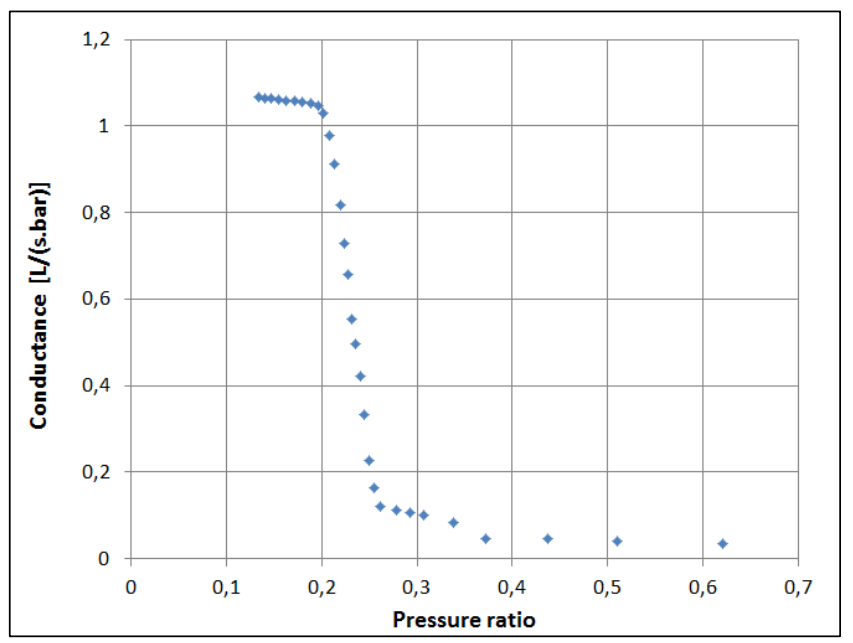

Figure 7: Conductance curve for the whole circuit (From $P_{U p}$ to $\left.P_{\text {Atm }}\right)$

\subsection{Dynamic behavior}

As the valve under study has a minimal pressure value in order to achieve its maximal displacement and consequently its maximal flow section, the dynamic tests have to be performed with upstream pressures above this value. In the following, the absolute working pressure is set to 0,7 MPa.

Once having the flow characteristics of the valve, the dynamic study can be performed. In this part the openingclosing frequency is controlled and the average mass flow rate in the circuit, the pressures, the solenoid current and the spool position are measured.

Having an electromagnetic actuator in the first stage of the valve, it is expected to obtain a set of well-known curves for the measured variables frequently present in the published works as in [8] where the characteristics of an 
electromagnetic flapper disk valve were investigated. Its switching time characteristics are presented in Figure 8.

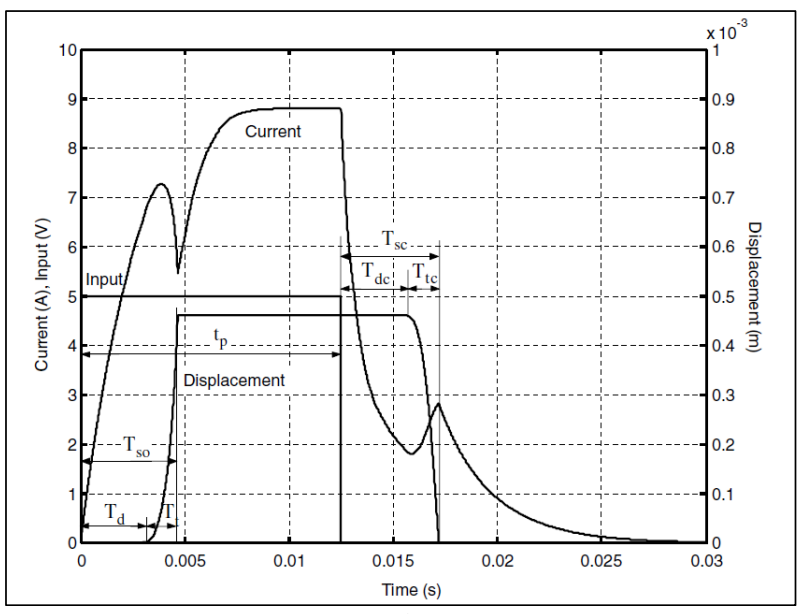

a)

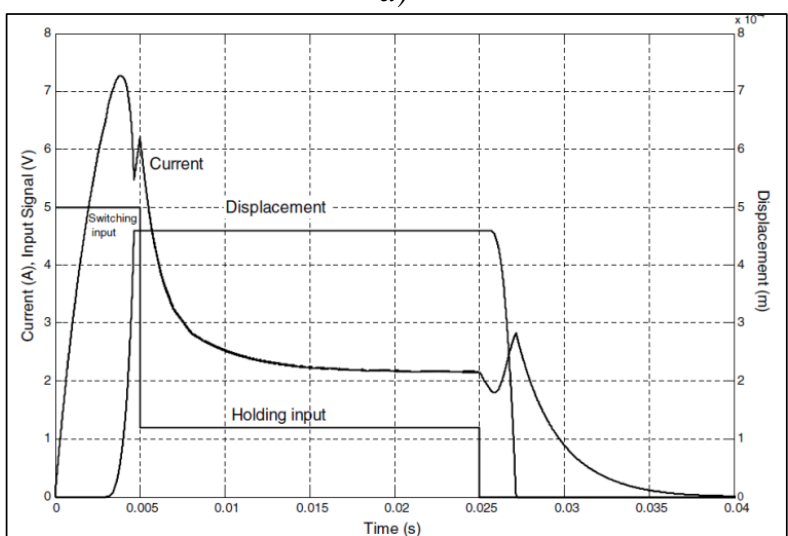

b)

Figure 8: a) Switching time characteristics ; b)Effects of holding current. [8]

In order to minimize the influence of the dynamic behavior of the electrical system on the final results, a different input was set to minimize the rising time of the current in the coil. In this way, the delayed time measured on opening is mostly due to the moving mass on the mechanical system. In the other hand, to accelerate the closing movement of the valve, a negative voltage is set in the coil. The input voltage profile is shown in Figure 9.

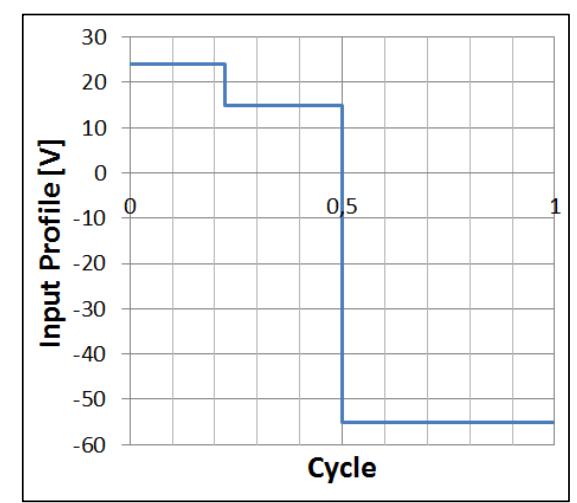

Figure 9: Input voltage profile per cycle
The dynamic tests were performed for frequencies from 10 $\mathrm{Hz}$ to $100 \mathrm{~Hz}$ with an increasing step of $10 \mathrm{~Hz}$. The results for a low frequency $(20 \mathrm{~Hz})$ and a higher frequency $(80 \mathrm{~Hz})$ are presented in Figure 10.

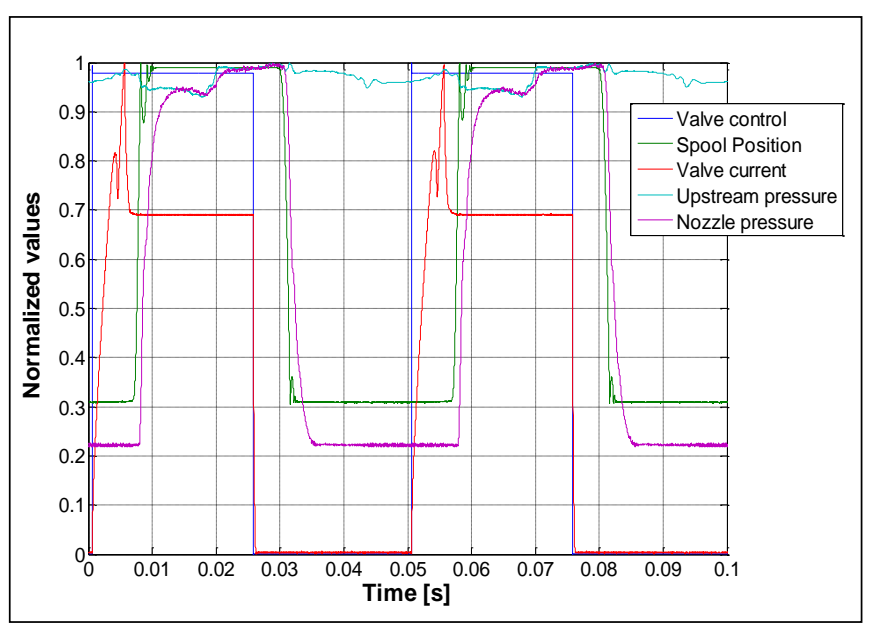

a)

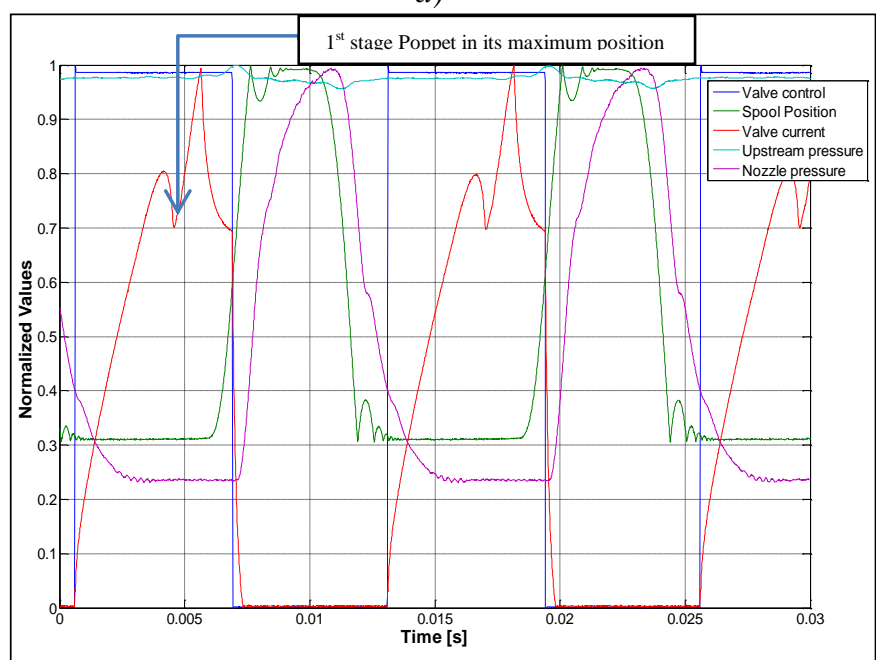

b)

Figure 10: Measurements for: a) $20 \mathrm{~Hz}$; b) $80 \mathrm{~Hz}$;

It is possible to notice a similar behavior to the one presented on Figure 8. For low frequencies (Figure 10a), the spool starts to move after a short delayed time and stays in its maximum position (opened) during almost the complete ON period of the input. The nozzle upstream pressure starts to increase after the spool starts to move and almost reaches the value of the upstream pressure, following its oscillations.

As the frequency is increased (Figure 10b), it is possible to observe that the spool starts to move almost the input is set to OFF and current starts to decrease. The electric system, even with the improvements in the input, spends a long time to establish the current in coil. In the other hand the valve has 2 stages with a pneumatic actuation, where the electromagnetic actuator moves the poppet of the first stage that reaches its maximum position at the point where the current has a local minimal value indicated in the Figure 10b. The delayed time from this point and the time where 
the spool starts to move is due to the pneumatic chamber being filled.

For frequencies higher than $120 \mathrm{~Hz}$ the spool cannot return to its initial position. Even with the poppet opening and closing normally, the pneumatic chamber does not have time to fill out, and the valve remains opened.

As the spool position and pressures along the circuit were acquired during the dynamic tests for the different frequencies and using the results of the steady state flow characterization, the instantaneous mass flow rate of the valve can be reconstructed for different frequencies by using equation 2 and the flow parameters of the valve shown in Figure 7. The results are shown in Figure 11 for one period and two frequencies.

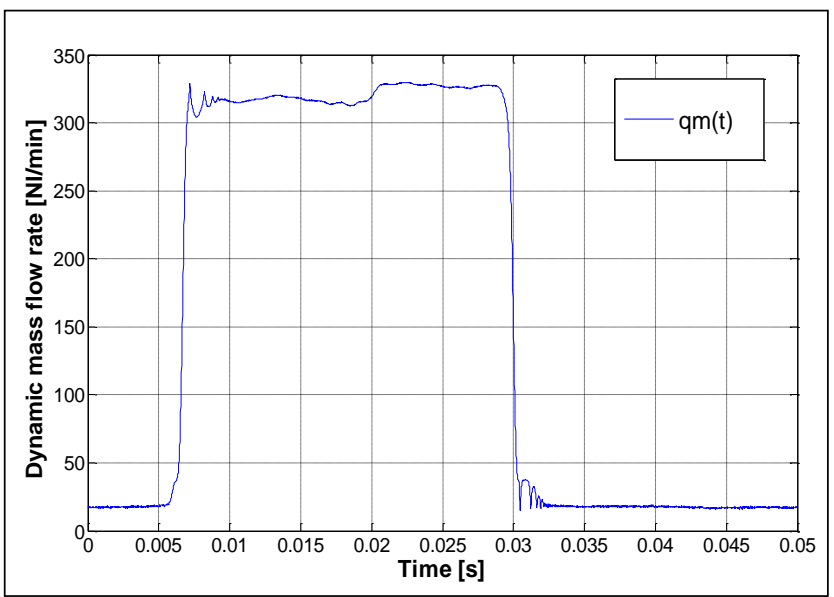

a)

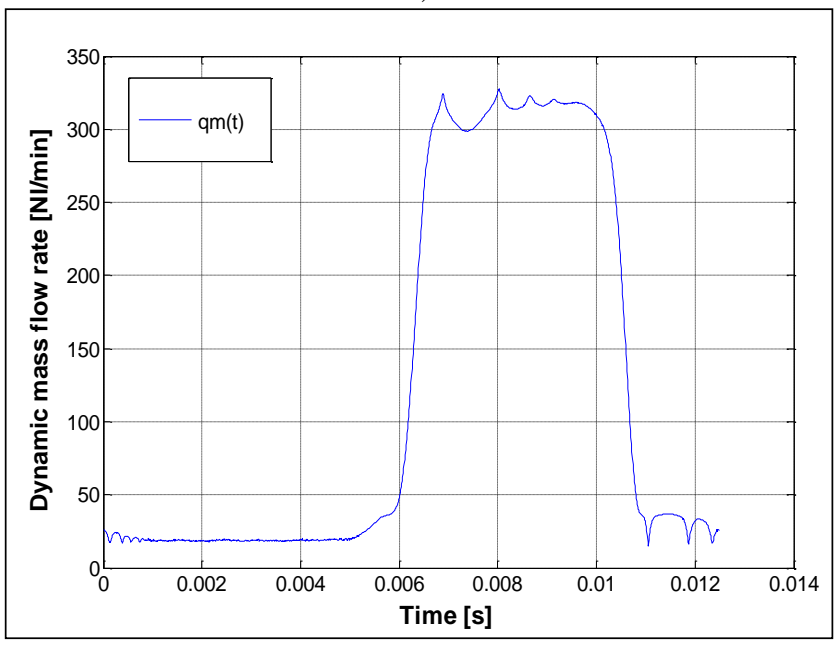

b)

Figure 11: Reconstruction of the dynamic mass flow rate for: a) $20 \mathrm{~Hz}$; b) $80 \mathrm{~Hz}$

By integrating the results obtained for each measurement during one cycle (duty cycle 50\%), it is possible to determine an average mass flow rate curve function of the frequencies and compare it with the average mass flow rate measured by the mass flowmeter. The measured and calculated values are presented in the Figure 12.

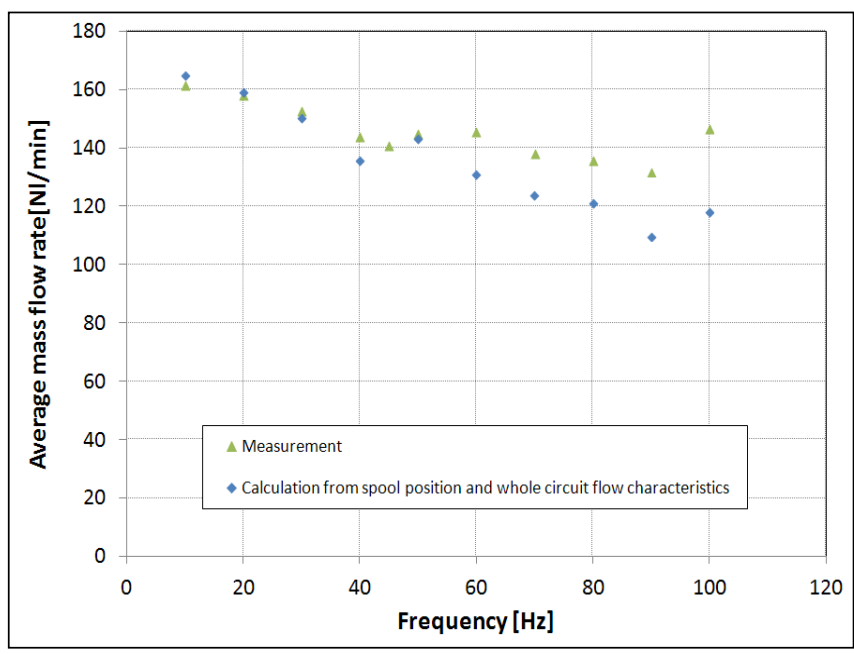

Figure 12: Average mass flow rate.

The results show a good parity for the estimated and measured average mass flow rate curves. For low frequencies up to $50 \mathrm{~Hz}$, the values have smaller discrepancies when compared to the higher ones. This is due to the fact the valve remains a longer time in its completely opened position, where its flow characteristics are wellknown, and also because this calculation has been conducted assuming the flow as instantaneously established. This is a correct approximation for low frequencies, but no longer for the higher ones where the transient effects are more important, which can be a source of errors. However, having the characteristics of the valve for some intermediate positions, and assuming a critical flow during the whole opening range of the valve, gives a good approximation for the average mass flow rate for high frequencies.

These transient effects will certainly be higher when using very fast switching valves. This is also the aim of this test bench to identify at which frequency the transitory effect of the flow establishment will become non negligible.

\subsection{Jet exploitation}

Another important part of the work consists in the characterization of the air jet coming from the system. As discussed before, the understanding of its characteristics in terms of velocity, pressure and temperature in the space and how they evolve in a dynamic operation of the system is crucial for the aim of optimizing the sorting operation.

Leonhard [9] presents the topology of the jet. Thus, it is known the jet has, at its origin, the same diameter as the nozzle orifice, which is $3 \mathrm{~mm}$ in the studied case. In this way, it is expected to have a jet with a conical geometry with an increasing diameter following its centerline and moving away from the nozzle.

For a first characterization of the jet, different parallel square measurement grids as shown in Figure 13a, perpendicular to the jet axis and spaced of $3 \mathrm{~mm}$, are set. 
These square grids have an increasing length as they distance the nozzle orifice in order to ensure the jet stays in the measuring zone.

However, as the probe used in this work is suitable for measurements in subsonic jets, the upstream pressure was reduced in order to prevent permanent damage to the probe.

The first validation tests with a steady state jet with the 5hole probe are presented in Figure 13.

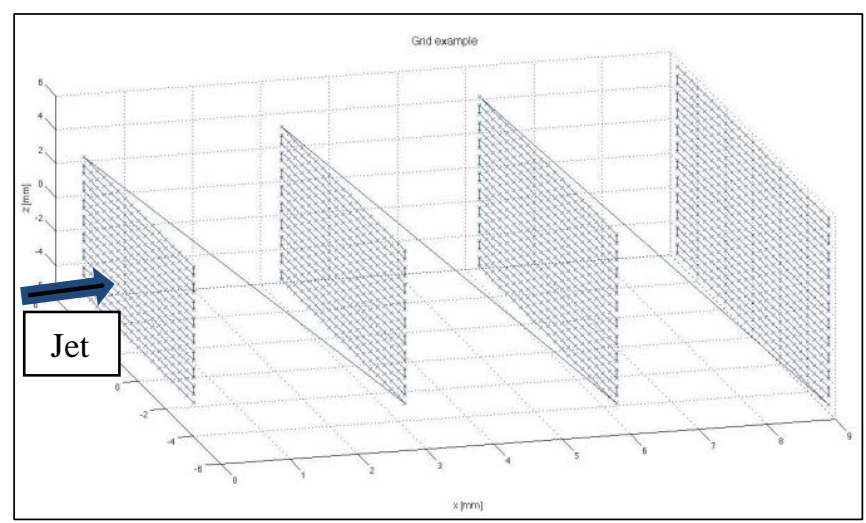

a)

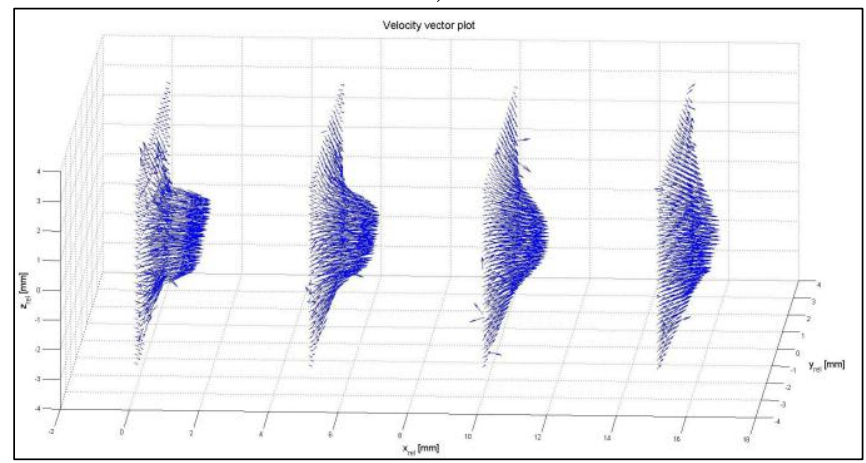

b)

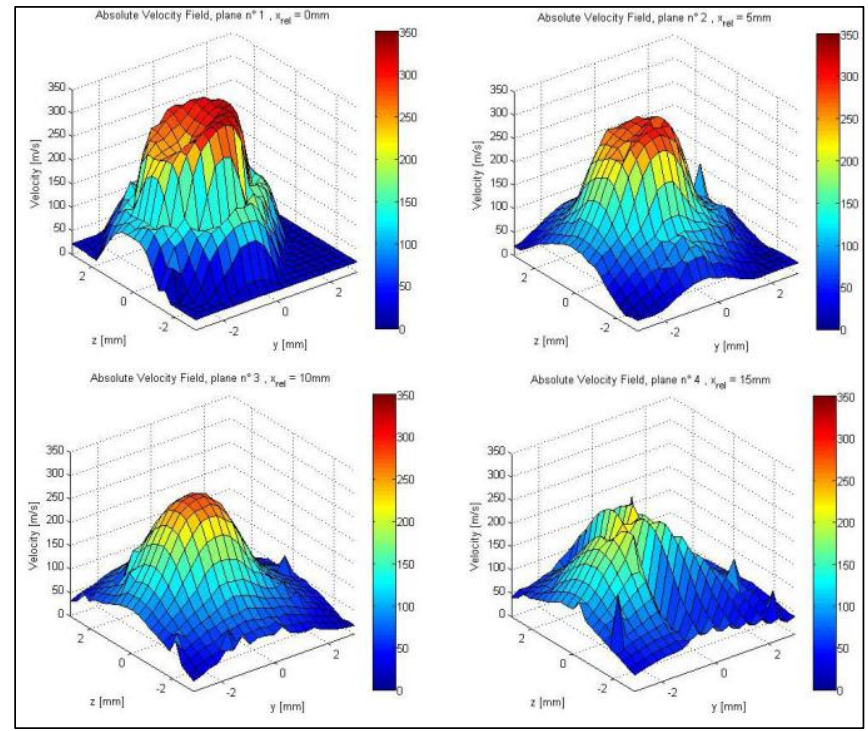

c)

Figure 13: a) Measurement grids; b) Normalized velocity vectors; c) Absolute Velocity Field
In Figure 13b the spatial evolution of the steady air jet is presented. In the plane close to the nozzle one can notice the potential core of the jet surrounded by a region where a mixing between the jet and the freestream air takes place. Inside this region, the jet keeps its maximum velocity magnitude, as well as pressure and temperature.

In a farther distance from the nozzle, the mixing region has spread inward and reaches the center line of the jet. In this point the diameter of the jet continues to increase and the potential core disappears.

A more detailed study of the jet in steady state and dynamic behavior is being carried out in order to determine the influence of the mechanical system on the jet generation. The goal is to identify the parameters which have more influence on the jet properties. In this way, one can optimize their system since the design of the elements (valve-nozzle and circuit between them) in order to have a more efficient sorting operation.

\section{Conclusion}

The development of the experimental bench as well as its instrumentation leads to a useful tool for the characterization of fast switching valves. By its use, it becomes possible to determine the flow characteristics of the valve without the need to disassembly the components and identify different phenomena that can occur along the inner and outer circuit from source to free air jet.

The possibility to drive the valve up to high frequencies and perform accurate measurement and analysis, allowed the determination of the working zone of the element in which the mechanical and pneumatic performance still achieve its nominal value suitable for the different applications.

The valve tested has a maximum operation frequency of 120 $\mathrm{Hz}$, which is still far from the faster valves already used in sorting applications. Thus, during tests with these faster elements, the transient effects will be more important and the need of a complete characterization of the elements shows to be a new challenge in order to reconstruct the average mass flow rate more accurately.

A first 3D analysis in terms of velocity of the air jet was performed in steady state and presented in this paper. The next step is to conduct a similar analysis in terms of pressure and temperature, and also in transient conditions to identify its dynamic behavior. This will enable to determine the different zones in the air jet in order to optimize the object sorting and evaluate the possible efficiency degradation of jets generated in high frequencies.

The future results could help the design of new valves taking into account the mechanical parameters which have more influence on the jet properties. This will deliver valves more adapted for sorting applications. 


\section{Nomenclature}

\begin{tabular}{|c|c|c|}
\hline Designation & Denotation & Unit \\
\hline$q_{m}$ & Mass flow rate & {$\left[\mathrm{m}^{3} / \mathrm{s}(\mathrm{ANR})\right]$} \\
\hline$P$ & Pressure & {$[\mathrm{Pa}]$} \\
\hline$P_{u}$ & $\begin{array}{l}\text { Upstream pressure of } \\
\text { element under } \\
\text { characterization }\end{array}$ & {$[\mathrm{Pa}]$} \\
\hline$P_{d}$ & $\begin{array}{l}\text { Downstream pressure } \\
\text { of the element under } \\
\text { characterization }\end{array}$ & {$[\mathrm{Pa}]$} \\
\hline C & Sonic conductance & {$\left[\mathrm{m}^{3} / \mathrm{s} / \mathrm{Pa}(\mathrm{ANR})\right]$} \\
\hline $\mathrm{Ce}$ & Conductance & {$\left[\mathrm{m}^{3} / \mathrm{s} / \mathrm{Pa}(\mathrm{ANR})\right]$} \\
\hline$T$ & Temperature & {$[\mathrm{K}]$} \\
\hline$T_{0}$ & $\begin{array}{l}\text { Temperature at } \\
\text { standard reference } \\
\text { atmosphere }(273.15)\end{array}$ & {$[\mathrm{K}]$} \\
\hline$\rho_{0}$ & $\begin{array}{l}\text { Mass density at } \\
\text { standard reference } \\
\text { atmosphere }(1,185)\end{array}$ & {$\left[\mathrm{kg} / \mathrm{m}^{3}\right]$} \\
\hline$b$ & Critical pressure ratio & - \\
\hline$T_{d}$ & $\begin{array}{l}\text { Delayed time to start } \\
\text { the opening movement }\end{array}$ & {$[\mathrm{s}]$} \\
\hline$T_{t}$ & Opening traveling time & {$[\mathrm{s}]$} \\
\hline$T_{\text {so }}$ & Opening switching time & {$[\mathrm{s}]$} \\
\hline$T_{s c}$ & Closing switching time & {$[\mathrm{s}]$} \\
\hline$t_{p}$ & Switching pulse time & {$[\mathrm{s}]$} \\
\hline$T_{d c}$ & $\begin{array}{l}\text { Delayed time to start } \\
\text { the closing movement }\end{array}$ & {$[\mathrm{s}]$} \\
\hline$T_{t c}$ & Closing traveling time & {$[\mathrm{s}]$} \\
\hline
\end{tabular}

\section{References}

[1] H. Yamada, S. Tsuchiya, T. Muto, Y. Suematsu. Development of a low cost high-speed on/off Digital valve using a bimorph PZT actuator. 4th JHPS International Symposium, 591-596, 1999.

[2] S.N. Yun, Y.B. Ham, J.H Park, D.Y. Ham, L.Y Lee. Pressure control characteristics of Electro-Pneumatic Regulator with Two multilayered PZT Valves. Proceeding to the 7th JFPS International Symposium on Fluid Power. 449-452. 2008.

[3] S.N. Yun, Y.B. Ham, J.H. Park, H.H. Kim, H.J So. Design Strategy of a Piezoelectric Valve for a Color Sorter. Journal of the Korean Physical Society, Vol. 57, No. 4, 913-917. 2010.
[4] J. Dubois. Etude expérimentale de jets libres compressibles ou en présence d'un obstacle. PhD Thesis. Marseille. 2010.

[5] C. Donaldson and R. Snedeker. A study of free jet impingement part 1: mean properties of free and impinging jets. Journal of fluid Mechanics, 45, Part 2. 281-319. 1971.

[6] D.T Branson, F.C Wang, D.N Johnston, D.G Tilley, C.R Bowen, P.S Keogh. Piezoelectrically actuated hydraulic valve design for high bandwidth and flow performance. Proc. IMechE Vol. 225(3). 345-359. 2011 .

[7] G.J Wilfong, M.A Bardorff, J.H Lumkes. Design and dynamics analysis of high speed ON/OFF Poppet Valves for digital pump/motors. Proc. $6^{\text {th }}$ FPNI-PhD Symposium. 259-269. 2010.

[8] E.E. Topçu, I. Yüksel, Z. Kamis. Development of electro-pneumatic fast switching valve and investigation of its characteristics. Journal Mechatronics 16. 365378. 2006

[9] A. Leonhard. Untersuchungen zum pneumatischen Sortieren von Schüttgütern mittels Freistrahlen. PhD Thesis. Dresden. 2010.

[10] Aeroprobe documentation.

[11] S.S. Truong. Conical Probe Calibration and Wind Tunnel Data Analysis of the Channeled Centerbody Inlet Experiment. Bachelor Thesis. California Polytechnic State University. 2011

[12] E.S. Johansen, O.K. Rediniotis, G. Jones. The Compressible Calibration of Miniature Multi-Hole Probes. Transactions of the ASME. Vol 123. 128-137. 2001.

[13]ISO Standard 6358. International standard, Pneumatic fluid Power Components using compressible Fluids Determination of flow rate characteristics. 1989.

[14] Kagawa, T., Cai, M., Kawashima, K., Wang, T., Nagaki, T., Hasegawa, T., and Oneyama, N. Extended representation of flow-rate characteristics for pneumatic components and its measurement using isothermal discharge method. Power Transmission and Motion Control, PTMC'04. 271-282. 2004. 